\title{
Optimization of Computations in Global Geopotential Field Applications
}

\author{
J.A.R. Blais ${ }^{1}$ and D.A. Provins ${ }^{2}$ \\ ${ }^{1,2}$ Department of Geomatics Engineering \\ ${ }^{1}$ Pacific Institute for the Mathematical Sciences \\ University of Calgary, Calgary, AB, T2N 1N4, Canada \\ blais@ucalgary.ca and provinsd@telusplanet.net
}

\begin{abstract}
Most boundary value problems of the geopotential field have integral and series solutions in terms of Green's convolution kernels. These solutions are advantageously evaluated using fast Spherical Harmonic Transforms (SHTs) for regular arrays of simulated or observed global data. However, the computational complexity and numerical conditioning of SHTs for relatively dense data are quite challenging and recent algorithmic developments warrant further investigations for geodetic and geophysical applications.

Global multiresolution applications for scalar, vector and tensor fields on the Earth and its neighborhood require spherical harmonic analysis and synthesis using convolution filters with data decimation and dilation. For global spherical grid applications, efficient and reliable SHTs are needed just as Fast Fourier Transforms (FFTs) are used in regional planar applications.

With the availability of enormous quantities of space, surface and subsurface data, extensive data structuring and management are unavoidable for most array computations. Different methodologies imply very different strategies and conflicting claims often appear in the literature. Discussions of the implicit and other assumptions with simulated results would undoubtedly help to clarify the situation and help decide on appropriate data structuring strategies for different computational applications.
\end{abstract}

\section{Introduction}

For every linear boundary value problem of the Earth's geopotential, it is possible to define a source or a Green's function. If this Green's function can be formulated explicitly, then the boundary value problem (BVP) is solved formally in terms of integral or series forms. In general, Green's function for a linear partial differential operator BVP is the solution for a Dirac delta impulse and homogeneous boundary conditions. Corresponding to the nonhomogeneous Dirichlet, Newmann and Robin BVPs of the Laplace operator, Green's functions for the sphere are well known with the solutions in integral and series forms readily available. Explicitly, the BVP solutions are expressed as convolutions of Green's functions and normal derivatives thereof with discrete measurements on or near the surface of the Earth.

Convolution operations are fundamental in linear filtering, solving BVPs using Green's functions and numerous other applications. In planar contexts, Fourier transform techniques are well established with FFTs, as the computational complexity 
is generally reduced from $\mathrm{O}\left(\mathrm{N}^{2}\right)$ to $\mathrm{O}(\mathrm{N} \operatorname{logN})$. In spherical contexts such as in geopotential applications, the situation is somewhat different because of the lack of some efficient SHTs for analysis, synthesis and convolution operations. Furthermore, as spherical displacements (or equivalently, spatial rotations) are generally non commutative, convolutions on the sphere are quite different from convolutions in the plane but can advantageously be evaluated using SHTs.

From a sampling perspective, it is often desirable to sample a band-limited function in such a way that the original function can be exactly recovered from the samples. In the case of functions on the real line, Shannon's sampling theorem states that a function whose Fourier transform has bounded support may be recovered from its samples provided that these are chosen uniformly at a rate of at least twice the bounding frequency. An important consideration is that the Fourier transform and hence the frequency spectrum can be computed from the discrete samples for analysis purposes.

For geodetic and geophysical applications, it is often desirable to sample a bandlimited function on the sphere so that the SHT can be efficiently evaluated as weighted sums of the samples. The investigation of numerical integration formulas has a long history and most relevant quadrature results on the sphere have drawbacks (see e.g. [16]). In the following, the primary objectives are for multiresolution applications using equiangular and Gaussian data grids for convolution and decimation/dilation operations in the study of geopotential BVPs (see e.g. [3] for more details). Brief comments about the asymptotic behavior of the Legendre functions of high degrees and orders are included.

Data structuring is becoming an important consideration with very large global datasets. Recently, Bond et al. [4] proposed a hierarchical structure known as an igloo pixelization for spherical harmonic transforms of the Cosmic Microwave Background (CMB) datasets. Another data structure known as HEALPix has been proposed by Wandelt et al. [22] for the same purposes. These data structuring strategies are quite different from the usual global approaches in geodesy and geophysics (e.g., [18]). Optimality in data structuring obviously depends on the intended applications.

\section{Sperical Harmonic Analysis and Synthesis}

The orthogonal or Fourier expansion of a function $f(\theta, \lambda)$ on the sphere $S^{2}$ is given by

$$
\mathrm{f}(\theta, \lambda)=\sum_{\mathrm{n}=0}^{\infty} \sum_{|m| \leq n} \mathrm{f}_{n, m} Y_{n}^{m}(\theta, \lambda)
$$

using polar angles $\theta$ and $\lambda$, where the basis functions $Y_{n}^{m}(\theta, \lambda)$ are called the spherical harmonics as $\Delta_{\mathrm{s}^{2}} \mathrm{Y}_{\mathrm{n}}^{\mathrm{m}}(\theta, \lambda)=0$, for all $|\mathrm{m}| \leq \mathrm{n}$ and $\mathrm{n}=0,1,2, \ldots$. This is an orthogonal decomposition in the Hilbert space $\boldsymbol{L}^{2}\left(\mathbf{S}^{2}\right)$ of functions square integrable with respect to the standard rotation invariant measure $d \sigma=\sin \theta d \theta d \lambda$ on $\mathbf{S}^{2}$. In particular, the Fourier or spherical harmonic coefficients appearing in the preceding expansion are obtained as inner products 


$$
\begin{aligned}
f_{n, m} & =\int_{s^{2}} f(\theta, \lambda) \bar{Y}_{n}^{m}(\theta, \lambda) d \sigma \\
& =\sqrt{\frac{(2 n+1)(n-m) !}{4 \pi(n+m) !}} \int_{S^{2}} f(\theta, \lambda) P_{n}^{m}(\cos \theta) e^{-i m \lambda} d \sigma
\end{aligned}
$$

where the $P_{n m}(\cos \theta)=(-1)^{m} P_{n}^{m}(\cos \theta)$ are the associated Legendre functions, with the overbar denoting the complex conjugate (e.g., [12]). In most practical applications, the functions $f(\theta, \lambda)$ are band-limited in the sense that only a finite number of those coefficients are nonzero, i.e. $f_{n, m} \equiv 0$ for all $n \geq N$.

The usual geodetic spherical harmonic formulation is slightly different with

$$
f(\theta, \lambda)=\sum_{n=0}^{\infty} \sum_{m=0}^{n}\left[\bar{C}_{n m} \cos m \lambda+\bar{S}_{n m} \sin m \lambda\right] \bar{P}_{n m}(\cos \theta)
$$

where

$$
\left\{\begin{array}{l}
\overline{\mathrm{C}}_{\mathrm{nm}} \\
\overline{\mathrm{S}}_{\mathrm{nm}}
\end{array}\right\}=\frac{1}{4 \pi} \int_{\mathrm{s}^{2}} \mathrm{f}(\theta, \lambda)\left\{\begin{array}{c}
\cos \mathrm{m} \lambda \\
\sin \mathrm{m} \lambda
\end{array}\right\} \overline{\mathrm{P}}_{\mathrm{nm}}(\cos \theta) \mathrm{d} \sigma
$$

and

$$
\begin{array}{rlrl}
\overline{\mathrm{P}}_{\mathrm{nm}}(\cos \theta) & =\sqrt{\frac{2(2 n+1)(\mathrm{n}-\mathrm{m}) !}{(n+m) !}} \mathrm{P}_{\mathrm{nm}}(\cos \theta) & & \text { for } \mathrm{m}=1, \ldots, \mathrm{n} \\
\overline{\mathrm{P}}_{\mathrm{n}}(\cos \theta) & =\sqrt{2 \mathrm{n}+1} \mathrm{P}_{\mathrm{n}}(\cos \theta) & \text { for } \mathrm{n}=0,1, \ldots
\end{array}
$$

in which the overbars refer to the usual geodetic normalization.

Geopotential models are fundamental in geodesy and geophysics. Figure 1 shows the geopotential model GPM98B of degree and order 1800 evaluated using software and coefficient dataset from Wenzel [23]. The latter geopotential model has been derived from the EGM96 model [10] through differential corrections, with integral formulas from $[15,25]$. From investigations of the numerical accuracy of the fully normalized Legendre functions, accuracy of the available software was limited to the degree and order indicated [24]. Denoting the above normalizing factor for $P_{n m}(\cos \theta)$ by $\mathrm{G}_{\mathrm{nm}}$, their magnitudes

$$
\mathrm{G}_{1800,1} \approx 0.047, \ldots . ., \mathrm{G}_{1800,1800} \approx 1.68 \times 10^{-5619}
$$

confirm that special care is required in the computations for meaningful numerical results.

For very high degree and order computations, asymptotic analyses of the behavior of the associated Legendre functions are required [21]. Considering the functions

$$
\psi_{\mathrm{n}}^{\mathrm{m}}(\theta)=\sqrt{\sin \theta} \mathrm{P}_{\mathrm{n}}^{\mathrm{m}}(\cos \theta)
$$

it can be shown that these satisfy the following simple Schrödinger differential equation

$$
\frac{d^{2}}{d \theta^{2}} \psi_{n}^{m}(\theta)=-\left[(n+1 / 2)^{2}-\left(m^{2}-1 / 4\right) \csc ^{2} \theta\right] \psi_{n}^{m}(\theta)
$$

which has the asymptotic solution behavior by the Wentzel-Kramers-Brillouin (WKB) method 


$$
\psi_{\mathrm{n}}^{\mathrm{m}}(\theta) \approx \exp \left\{\mathrm{i} \int^{\theta}\left[(\mathrm{n}+1 / 2)^{2}-\left(\mathrm{m}^{2}-1 / 4\right) \csc ^{2} \mathrm{t}\right]^{1 / 2} \mathrm{dt}\right\}
$$

when the square root is real in the integrand. The behavior of $\psi_{n}^{m}(\theta)$ is oscillatory with very large magnitudes near 0 and $\pi$ (see Figure 2 for plots of $\mathrm{P}_{60}^{20}(\cos \theta)$ and $\left.\psi_{60}^{20}(\theta)\right)$. Mohlenkamp [12] has reformulated the spherical analysis and synthesis in terms of these functions with advantageous computational results.

\section{Discretization and Numerical Analysis}

Colombo [5] has discretized the preceding geodetic formulation with $\theta_{\mathrm{j}}=\mathrm{j} \pi / \mathrm{N}, \mathrm{j}=1$, $2, \ldots, \mathrm{N}$ and $\lambda_{\mathrm{k}}=\mathrm{k} \pi / \mathrm{N}, \mathrm{k}=1,2, \ldots, 2 \mathrm{~N}$. This is only an approximate quadrature approach that uses summations to estimate the numerical integrals for the frequency spectrum and the computational effort required is $\mathrm{O}\left(\mathrm{N}^{4}\right)$, without counting the computations involved in the evaluation of the Legendre functions. The longitude computations can easily be carried out using FFTs and the corresponding computational effort is reduced to $\mathrm{O}\left(\mathrm{N}^{3} \log \mathrm{N}\right)$.

Legendre quadrature is well known to provide an exact representation of polynomials of degrees up to $2 \mathrm{~N}-1$ using only $\mathrm{N}$ data values at the zeros of the Legendre polynomials. Driscoll and Healy [7] have exploited these quadrature ideas in an exact algorithm for a reversible SHT using the following $\mathrm{N}^{2}$ data grid for degree and order N:

For synthesis purposes, given a band-limited square integrable function $f(\theta, \lambda)$, with co-latitude $\theta$ and longitude $\lambda$, given normalized spherical harmonic coefficients $a_{n m}$ and $b_{n m}$ for $m \leq n, n=0,1,2, \ldots, N-1$,

$$
\begin{aligned}
f(\theta, \lambda) & =\sum_{n=0}^{N-1} \sum_{m=0}^{n}\left(a_{n m} \cos m \lambda+b_{n m} \sin m \lambda\right) P_{n m}(\cos \theta) \\
& =\sum_{m=0}^{N-1} \sum_{n=m}^{N-1}\left(a_{n m} \cos m \lambda+b_{n m} \sin m \lambda\right) P_{n m}(\cos \theta) \\
& =\sum_{m=0}^{N-1}\left\{\left(\sum_{n=m}^{N-1} a_{n m} P_{n m}(\cos \theta)\right) \cos m \lambda+\left(\sum_{n=m}^{N-1} b_{n m} P_{n m}(\cos \theta)\right) \sin m \lambda\right\}
\end{aligned}
$$

and defining

$$
A_{m}(\theta)=\sum_{n=m}^{N-1} a_{n m} P_{n m}(\cos \theta)
$$

and

one has

$$
B_{m}(\theta)=\sum_{n=m}^{N-1} b_{n m} P_{n m}(\cos \theta)
$$




$$
\begin{aligned}
f(\theta, \lambda) & =\sum_{m=0}^{N-1}\left\{A_{m}(\theta) \cos m \lambda+B_{m}(\theta) \sin m \lambda\right\} \\
& =\frac{1}{2} \sum_{m=0}^{N-1}\left\{\left[A_{m}(\theta)+i B_{m}(\theta)\right] e^{-i m \lambda}+\left[A_{m}(\theta)-i B_{m}(\theta)\right] e^{i m \lambda}\right\} \\
& \left.=\frac{1}{2}\left\{\operatorname{DFT}\left[A_{m}(\theta)+i B_{m}(\theta)\right]+\overline{\operatorname{DFT}\left[A_{m}(\theta)+i B_{m}(\theta)\right.}\right]\right\} \\
& =\operatorname{ReDFT}\left[A_{m}(\theta)+i B_{m}(\theta)\right]
\end{aligned}
$$

assuming discrete longitudes $\lambda_{\mathrm{k}}=2 \pi \mathrm{k} / \mathrm{N}, \mathrm{k}=0,1,2, \ldots, \mathrm{N}-1$, with the Discrete Fourier Transform (DFT), for discrete co-latitudes $\theta$ to be determined. Writing $C_{m}(\theta)=A_{m}(\theta)+i B_{m}(\theta)$ and $c_{n m}(\theta)=a_{n m}(\theta)+i b_{n m}(\theta)$, one then has

$$
\mathrm{f}\left(\theta, \lambda_{\mathrm{k}}\right)=\operatorname{Re} \operatorname{DFT}\left[\mathrm{C}_{\mathrm{m}}(\theta)\right]
$$

where

$$
C_{m}(\theta)=\sum_{n=m}^{N-1}\left(a_{n m}+i b_{n m}\right) P_{n m}(\cos \theta)=\sum_{n=m}^{N-1} c_{n m} P_{n m}(\cos \theta)
$$

that is,

$$
\begin{aligned}
& \mathrm{C}_{0}(\theta)=\mathrm{c}_{00} \mathrm{P}_{00}(\cos \theta)+\mathrm{c}_{10} \mathrm{P}_{10}(\cos \theta)+\mathrm{c}_{20} \mathrm{P}_{20}(\cos \theta)+\ldots+\mathrm{c}_{\mathrm{N}-1,0} \mathrm{P}_{\mathrm{N}-1,0}(\cos \theta) \\
& \mathrm{C}_{1}(\theta)=\mathrm{c}_{11} \mathrm{P}_{11}(\cos \theta)+\mathrm{c}_{21} \mathrm{P}_{21}(\cos \theta)+\mathrm{c}_{31} \mathrm{P}_{31}(\cos \theta)+\ldots+\mathrm{c}_{\mathrm{N}-1,1} \mathrm{P}_{\mathrm{N}-1,1}(\cos \theta) \\
& \mathrm{C}_{2}(\theta)=\mathrm{c}_{22} \mathrm{P}_{22}(\cos \theta)+\mathrm{c}_{32} \mathrm{P}_{32}(\cos \theta)+\mathrm{c}_{42} \mathrm{P}_{42}(\cos \theta)+\ldots+\mathrm{c}_{\mathrm{N}-1,2} \mathrm{P}_{\mathrm{N}-1,2}(\cos \theta)
\end{aligned}
$$

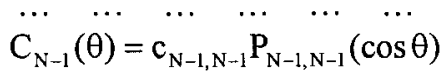

for discrete co-latitudes $\theta$ to be determined.

For analysis, given a band-limited square integrable function $f(\theta, \lambda)$, with colatitude $\theta$ and longitude $\lambda$, with observations at $\lambda_{\mathrm{k}}=2 \pi \mathrm{k} / \mathrm{N}$ and $\theta_{\mathrm{j}}=(\mathrm{j}+1 / 2) \pi / \mathrm{N}, \mathrm{k}$, $\mathrm{j}=0,1,2, \ldots, \mathrm{N}-1$, assuming $\mathrm{N}$ to be a power of 2 , using Driscoll and Healy's formulation [7], the normalized spherical harmonic coefficients $a_{n m}$ and $b_{n m}$ for $m \leq$ $\mathrm{n}, \mathrm{n}=0,1,2, \ldots, \mathrm{N}-1$, can be evaluated as follows:

$$
\left\{\begin{array}{l}
a_{n m} \\
b_{n m}
\end{array}\right\}=\frac{2 \pi(-1)^{m}}{N} \sum_{j=0}^{N-1} \sum_{k=0}^{N-1} d_{j} f\left(\theta_{j}, \lambda_{k}\right)\left\{\begin{array}{l}
\cos m \lambda_{k} \\
\sin m \lambda_{k}
\end{array}\right\} P_{n m}\left(\cos \theta_{j}\right)
$$

or

$$
\begin{aligned}
c_{n m} & =\frac{2 \pi(-1)^{m}}{N} \sum_{j=0}^{N-1} \sum_{k=0}^{N-1} d_{j} f\left(\theta_{j}, \lambda_{k}\right) e^{+i m \lambda_{k}} P_{n m}\left(\cos \theta_{j}\right) \\
& =\frac{2 \pi}{N} \sum_{j=0}^{N-1} \sum_{k=0}^{N-1}(-1)^{m} d_{j} f\left(\theta_{j}, \lambda_{k}\right) e^{+i m \lambda_{k}} P_{n m}\left(\cos \theta_{j}\right) \\
& =\frac{2 \pi}{N} \sum_{j=0}^{N-1}(-1)^{m} d_{j} P_{n m}\left(\cos \theta_{j}\right) \operatorname{IDFT}_{k}\left[f\left(\theta_{j}, \lambda_{k}\right)\right]
\end{aligned}
$$

with the Inverse Discrete Fourier Transform (IDFT), where the quadrature weights

$$
d_{j}=\frac{2 \sqrt{2}}{N} \sin [(j+1 / 2) \pi / N] \sum_{h=0}^{N / 2-1} \frac{1}{2 h+1} \sin [(2 h+1)(j+1 / 2) \pi / N]
$$


assuming the usual normalization of the Legendre functions. In practice, DFTs and IDFTs would of course be replaced by FFTs and IFFTs for efficiency. The offsetting of the co-latitudes $\theta_{\mathrm{j}}$ is to avoid the unacceptable situations at the poles for geocomputations. Other formulations such as the SpherePack software [1] require that both poles be included with an equiangular grid or a Gaussian grid. Mohlenkamp [12] uses a Gaussian grid for better accuracy.

Other authors have used "igloo-like" and "equal-area, iso-latitude" constructions to facilitate spherical harmonic transforms and other operations on the sphere (see, e.g. $[13,6,9])$. In particular, the latter construction has been used for fast Haar wavelet transforms.

Linear convolutions are among the most fundamental operations in digital signal processing. They correspond to the filtering operation of a (data) sequence by another sequence that characterizes the filter. Considering square integrable functions over the sphere, i.e. $\boldsymbol{L}^{2}\left(\mathbf{S}^{2}\right)$, a convolution algebra of spherical harmonic polynomials can be obtained, but as translations in the plane correspond to rotations on the sphere which do not always commute, the corresponding convolution algebra is non commutative or non Abelian. Using the SHTs for convolution equation $\mathrm{Z}(\mathrm{P})=\mathrm{X}(\mathrm{P}) * \mathrm{Y}(\mathrm{P})$ for points $\mathbf{P}$ on the sphere $\mathbf{S}^{2}$,

$$
Z_{n, m}=\frac{4 \pi}{2 n+1} X_{n} Y_{n, m} \neq \frac{4 \pi}{2 n+1} Y_{n} X_{n, m}
$$

with the inequality due to the non-commutativity of displacements on the sphere, where as above, $\mathbf{n}$ denotes the degree and $\mathrm{m}$, the order in the SHTs. Examples of convolutions on the sphere and in neighboring space are given in [3], mainly for global multiresolution applications.

\section{Data Stucturing Considerations}

Enormous quantities of space, surface and subsurface data imply extensive data structuring and management requirements for most array computations. For some years, various methods have been proposed for data analysis, storage and visualization [e.g. 2, 11, 8, 26]. More recently, attempts have been made to address the data management and processing problems. The Hierarchical Data Format (HDF) file organization has recently been promoted for many processing tasks and discipline areas [20]. HDF's limitations have largely been overcome in Version 5 by employing parallelism in data storage and computation [14].

The use of spherical quadtrees has been proposed by NASA to solve storage, multiple resolution analysis and to improve interactive browsing [17]. For the analysis of the Cosmic Microwave Background (CMB), both the igloo [6] and HEALPix [9] approaches have been proposed. The latter is said to meet the needs for a hierarchical database structure, an equal area pixelization and an isolatitude distribution of area elements. A software package that implements this discretization and provides the means of performing fast spherical harmonic transforms is available. The igloo approach gives an exact azimuthal symmetry at each latitude and hence exact spherical harmonic transforms are possible. 


\section{Concluding Remarks}

Spherical computations involve analysis and synthesis using SHTs for convolutions in linear filtering, boundary value problems and numerous other applications. With discrete data on regular spherical grids, different quadrature formulations exist in the literature and different conventions make the intercomparative analyses quite challenging. Different strategies imply different grids such as equiangular, Gaussian, etc. and the normalization used in the resulting frequency spectra is often different.

Considerable work has been done on solving the computational complexities, and enhancing the speed of calculation of spherical harmonics. The approach of Driscoll and Healy [7] is exact for exact arithmetic, while the earlier approach by Swarztrauber [19] which was a quadrature method as well, also provided an exact analysis for a continuous function expressible in terms of a discrete surface harmonic basis. The latter method was quite accurate, with cited errors of the order of $10^{-13}$ for a 2.5 degree grid. Both methods provide recovery of the original signal provided it is not aliased in the sampling process.

With respect to gridding, both Gaussian quadrature and equiangular grids have been used to obtain accurate results. In particular, the SpherePack code, which in part is based on Swarztrauber's work, provides both grids, and the mechanism to move between the two. More recently, Mohlenkamp [12] has developed the means to expand a function on a Gaussian grid in $\mathrm{O}\left(\mathrm{N}^{2} \log ^{2} \mathrm{~N}\right)$ time, although the speed is said to depend in part on the use of pre-computed and compressed representations of the associated Legendre function bases. More optimization research is clearly warranted for fast SHTs to be comparable to two-dimensional FFTs.

\section{Acknowledgements.}

The authors would like to acknowledge the sponsorship of the Natural Science and Engineering Research Council in the form of a Research Grant to the first author on Computational Tools for the Geosciences.

\section{References}

1. Adams, J.C. and P.N. Swarztrauber. SPHEREPACK 2.0: A Model Development Facility, 1997. http://www.scd.ucar.edu/softlib/SPHERE.html

2. Augenbaum, J.M. and C.S. Peskin. On the Construction of the Voronoi Mesh on a Sphere. Journal of Computational Physics, 59, pp. 177-192, 1985.

3. Blais, J.A.R. and D.A. Provins. Spherical Harmonic Analysis and Synthesis for Global Multiresolution Applications. Journal of Geodesy, vol.76, no.1, pp.29-35, 2002.

4. Bond, J.R., R.G. Crittenden, A.H. Jaffe and L. Knox. Computing Challenges of the Cosmic Microwave Background. Computing in Science and Engineering, March-April, pp.21-35, 1999.

5. Colombo, O. Numerical Methods for Harmonic Analysis on the Sphere. Ohio State University, Report no. 310, 1981.

6. Crittenden, R.G. and N.G. Turok. Exactly Azimuthal Pixelizations of the Sky, 1998. http://xxx.lanl.gov/list/astro-ph/9806[374] (I.e. article 374 in the 9806 directory). 
7. Driscoll, J.R. and D.M. Healy, Jr. Computing Fourier Transforms and Convolutions on the 2-Sphere. Advances in Applied Mathematics, 15, pp. 202-250, 1994.

8. Gold, C.M. Problems with Handling Spatial Data - The Voronoi's Approach. CISM Journal ACSGC, vol. 45, no. 1, pp. 65-80, 1991.

9. Gorski, K.M., E. Hivon and B.D. Wandelt. Analysis Issues for Large CMB Data Sets. Proceedings: Evolution of Large Scale Structure, Garching, 1998.

10. Lemoine, F.G., D.E. Smith, L. Kunz, R. Smith, E.C. Pavlis, N.K. Pavlis, S.M. Klosko, D.S. Chinn, M.H. Torrence, R.G. Williamson, C.M. Fox, K.E. Rachlin, Y.M. Wang, S.C. Kenyon, R. Salman, R. Trimmer, R.H. Rapp and R.S. Nerem. The Development of the NASA GSFC and NIMA Joint Geopotential Model. International Symposium Gravity, Geoid and Marine Geodesy, Tokyo, International Association of Geodesy Symposia, Vol. 117, pp. 461-469, Springer-Verlag, 1996.

11. Lukatela, H. Hipparchus Geopositioning Model: An Overview. Proceedings of AUTOCARTO 8, 1987. http://www.geodyssey.com.

12. Mohlenkamp, M.J. A Fast Transform for Spherical Harmonics. The Journal of Fourier Analysis and Applications, vol. 5, nos. 2/3, pp. 159-184, 1999.

13. Muciaccia P.F., P. Natoli and N. Vittorio. Fast Spherical Harmonic Analysis: A Quick Algorithm for Generating and/or Inverting Full-Sky, High Resolution Cosmic Microwave Background Anisotropy Maps. The Astrophysical Journal, 488, pp. L63-L66, 1997.

14. NCSA Introduction to HDF5 Release 1.0, National Center for Supercomputing Applications, 1999.

15. Paul, R.H. Recurrence Relations for the Integrals of Associated Legendre Functions. Bulletin Géodésique, Vol. 52, pp. 177-190, 1978.

16. Ricardi, L.J. and M.L. Burrows. A Recurrence Technique for Expanding a Function in Spherical Harmonics. IEEE Transactions on Computers, June, pp. 583-585, 1972.

17. Short, N.M., Jr., R.F. Cromp, W.J. Campbell, J.C. Tilton, J.L. LeMoigne, G. Fekete, N.S. Netanyahu and G. Sylvain. AI Challenges within NASA's Mission to Planet Earth. Workshop Notes for the 1994 National Conf. on Artificial Intelligence, C.L. Mason (Ed.).

18. Sneeuw, N. Global Spherical Harmonic Analysis by Least-Squares and Numerical Quadrature Methods in Historical Perspective. Geophys. J. Int. (1994) 118, 707-716.

19. Swarztrauber, P.N. On the Spectral Approximation of Discrete Scalar and Vector Functions on the Sphere. SIAM Journal of Num. Analysis, Vol.16, No. 6, pp. 934-949, 1979.

20. Tan, C.J., J.A.R. Blais and D.A. Provins. Large Imagery Data Structuring Using Hierarchical Data Format for Parallel Computing and Visualization. High Performance Computing Systems and Applications, edited by A. Pollard, D. J.K. Mewhort and D.F. Weaver, Kluwer Academic Publishers, Chapter 39, pp. 371-386, 2000.

21. Varshalovich, D.A., A.N. Moskalev and V.K. Khersonskij. Quantum Theory of Angular Momentum. World Scientific Publishing, Singapore, 1988.

22. Wandelt, B.D., E. Hivon and K.M. Gorski. Topological Analysis of High-Resolution CMB Maps. Theoretical Astrophysics Centre, Copenhagen, Denmark, 1998, 4 pages.

23. Wenzel, G. Ultra High Degree Geopotential Model GPM3E97A to Degree and Order 1800 Tailored to Europe. Proceedings of the Second Continental Workshop on the Geoid in Europe, Budapest, 1998.

24. Wenzel, G. Ultra High Degree Geopotential Models GPM98A, B and C to Degree 1800. Preprint, Bulletin of International Geoid Service, Milan, 1998.

25. Wenzel, G. Hochaufloesende Kugelfunktionsmodelle fuer das Gravitationspotential der Erde. Wissenschaftliche Arbeiten der Fachrichtung Vermessungswesen der Universitat Hannover, Nr. 135, Hannover, 1985.

26. Zheng, C., J. Nie and J.A.R. Blais. Applicability of the Hipparchus Software in Geoscience Information Systems. Proceedings of the Canadian 1994 GIS Conference in Ottawa, pp. 434-442. 


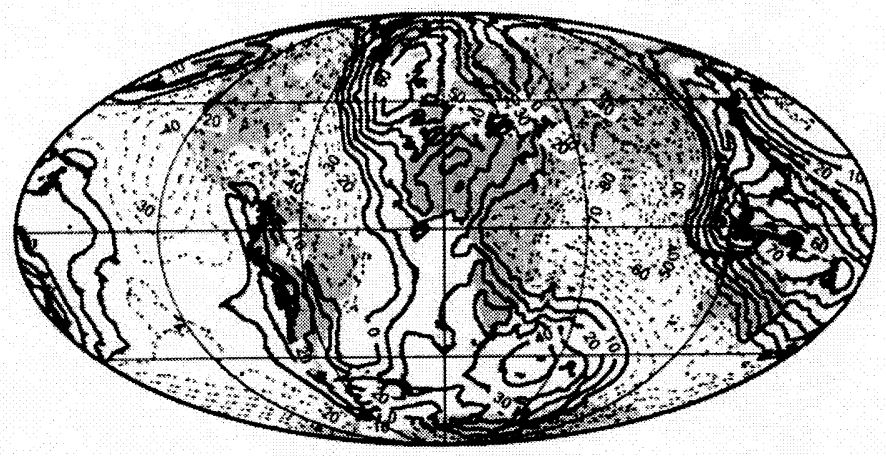

Fig.1. GPM 98 B Undulation Map of Degree and Order 1800.
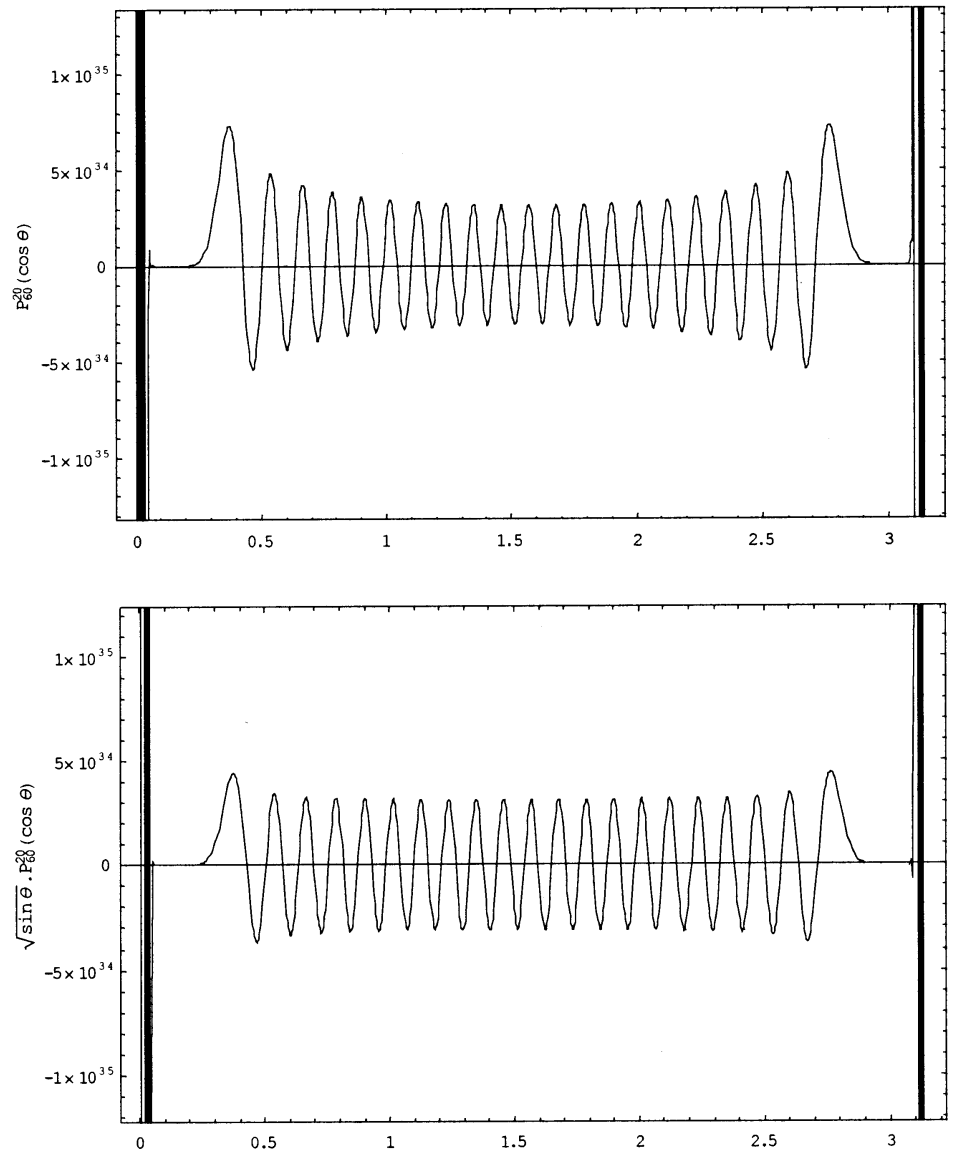

Fig. 2. Plots of $\mathrm{P}_{60}^{20}(\cos \theta)$ and $\psi_{60}^{20}(\theta) \equiv \sqrt{\sin \theta} \mathrm{P}_{60}^{20}(\cos \theta), \quad 0 \leq \theta \leq \pi$. 